\title{
MONITORING OF EXTRAGALACTIC RADIO SOURCES VIA GEODETIC VLBI OBSERVATIONS
}

\author{
Guoqiang Tang and Bernt Rönnäng \\ Onsala Space Observatory \\ Chalmers University of Technology \\ S-439 00 Onsala \\ Sweden
}

\section{INTRODUCTION}

It was pointed out already in the early stage of the development of the Mark-III VLBI that the structure of the quasars could affect VLBI results for geodesy or astrometry (Cotton, 1980; Robertson, 1981). As errors from other sources are gradually reduced, this effect can not be ignored any longer. We should monitor the structure variations of the sources and study their internal kinematics closely. $8 \mathrm{GHz}$ maps made from geodetic VLBI observations may give us important information.

\section{MULTI-EPOCH MAPS AT $8 \mathrm{GHz}$}

Mark-III geodetic VLBI observations are used to map some compact extragalactic radio sources. Our maps are made from global geodetic VLBI experiments, e.g. MERIT campaign, CDP and IRIS projects etc., in which Onsala is involved. Six maps of 4 C39.25 from 1980-1985 have been made(Tang, Rönnäng and Bååth, 1987). In our maps, a third component became visible since 1984.30. The component B moves towards $\mathrm{A}$ (or vise versa) at an apparent projected velocity of $\mathrm{v} / \mathrm{c}=3.1 \pm 0.4\left(\mathrm{z}=0.699, \mathrm{q}_{0}=0.05\right.$ and $\mathrm{H}_{0}=100 \mathrm{~km} / \mathrm{s} / \mathrm{Mpc}$ ). Five maps of $3 \mathrm{C} 345$ at $8 \mathrm{GHz}$ are obtained from the same geodetic VLBI experiments. The maps show the characteristics of this most well-studied quasar. The component C3.5(Biretta et al, 1986) is confirmed in the map of 1982.96. The expansions of jets C3, C4 and C5 from the core are clearly indicated.

\section{CALCULATIONS OF SOURCE STRUCTURE EFFECTS ON GEODETIC VLBI}

The group delay correction due to the radio source structure is given by,

$$
T_{\text {str }}=\frac{\operatorname{Re}(\mathrm{V}) \frac{\partial}{\partial w}[\operatorname{Im}(\mathrm{V})]-\operatorname{Im}(\mathrm{V}) \frac{\partial}{\partial w}[\operatorname{Re}(\mathrm{V})]}{|\mathrm{V}|^{2}}
$$

where the visibility $\mathrm{V}(\mathrm{u}, \mathrm{v})$ is the Fourier transform of the source brightness distribution $\mathrm{B}(\mathrm{x}, \mathrm{y})$, which can be properly represented by a number of Gaussians. The Gaussian models are obtained from X-band hybrid maps made from geodetic VLBI data.

The delay corrections on the baseline Onsala-Westford due to the structure of 3C 345 and 4C39.25 are calculated during the CDP experiments 15-18 December 1982 (Fig.1). It is shown that the delay correction 431

M. J. Reid and J. M. Moran (eds.), The Impact of VLBI on Astrophysics and Geophysics, 431-432.

(C) 1988 by the IAU. 


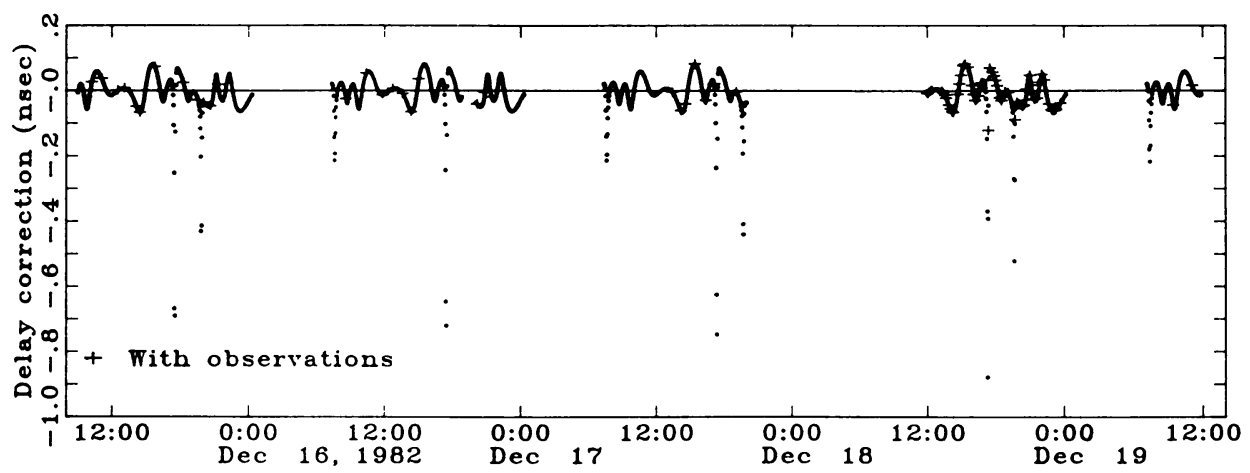

Fig. 1-1

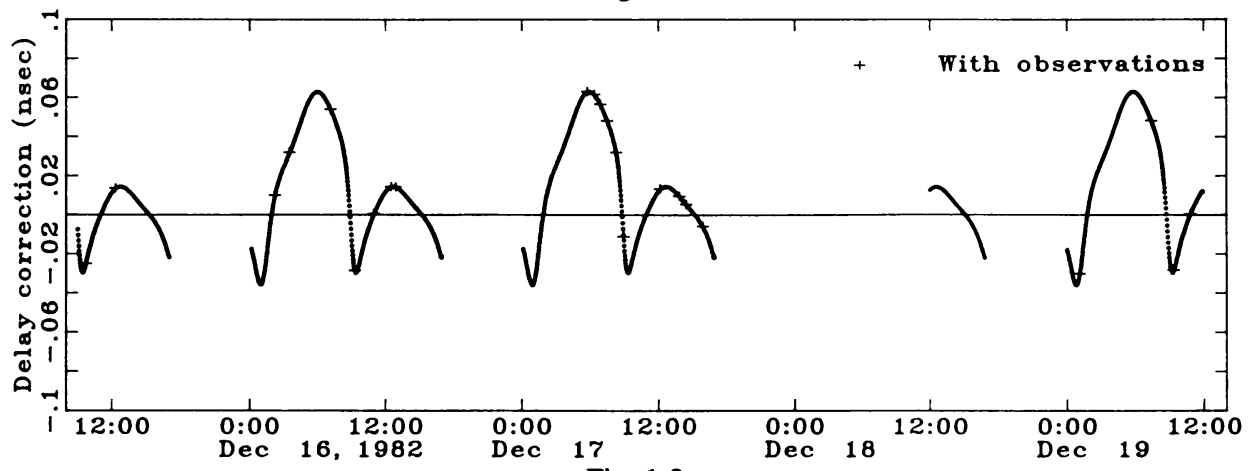

Fig. 1-2

Figure 1. The group delay corrections due to the radio source strucure on the Westford-Onsala baseline during the global geodetic VLBI experiment, Dec. 15-18, 1982. The calculations are based on the Gaussian models obtained from the $8 \mathrm{GHz}$ maps which are made from the same observations. Fig. 1-1 shows the structure effect of $3 \mathrm{C} 345$, and Fig. 1-2 shows that of $4 \mathrm{C} 39.25$.

due to the source structure varys periodically from a few hours to daily. In general, this error effect falls in the range of current measuring accuracy $( \pm 3 \mathrm{~cm})$ of geodetic VLBI. However, for some large scale one-sided core-jet sources, it can change dramatically.

Hybrid maps made from geodetic VLBI data can provide valuable information on the internal kinematics of compact extragalactic radio sources and they are certainly important for estimation of the source structure effect on geodetic VLBI. Source monitoring will become more significant if it is based on a long term basis. Therefore the mapping will be continued and expanded. Further investigations are needed to study the source structure effect on geodetic VLBI, especially the long term influence, using source maps obtained from geodetic VLBI observations.

\section{REFERENCES}

Biretta, J.A., Moore, R.L. and Cohen, M.H.:1986, Astrophys. J., 308, 93.

Cotton, W.E.:1980, in Radio Interferometry Jechniques for Geodesy, NASA Conference Publication, No.2115 (NASA, Washington, DC), pp.193-198.

Robertson, D.S.:1981, in Reference Coordinate Systems for Earth Dynamics, eds. E.M. Gaposchkin and B. Kolaczek (Reidel, Dordrecht), pp.205-216.

Tang, G., Rőnnăng, B. and Båăth, L.:1987, Astron. Astrophys. (in press). 